\title{
Production of $\eta$ mesons in nucleon-nucleon collisions
}

\author{
V. Baru ${ }^{a, b}$, A.M. Gasparyan ${ }^{a, b}$, J. Haidenbauer ${ }^{a}$, C. Hanhart ${ }^{a}$, A.E. Kudryavtsev ${ }^{b}$, and J. \\ $\operatorname{Speth}^{a}$ \\ ${ }^{a}$ Institut für Kernphysik, Forschungszentrum Jülich GmbH, \\ D-52425 Jülich, Germany \\ ${ }^{b}$ Institute of Theoretical and Experimental Physics, \\ 117259, B. Cheremushkinskaya 25, Moscow, Russia
}

\begin{abstract}
A microscopic calculation of near-threshold $\eta$-meson production in the reaction $N N \rightarrow N N \eta$ is presented. It is assumed that the $\eta$ meson is produced via direct emission and via elementary rescattering processes $M N \rightarrow \eta N$ of various mesons $M=\pi, \rho$, etc. As a novel feature the amplitudes for the latter production mechanism are taken from a multi-channel meson-exchange model of the $\pi N$ system developed by the Jülich group which contains explicitly the channels $\pi N, \rho N, \eta N, \sigma N$, and $\pi \Delta$. Furthermore, effects of the $N N$ interaction in the final as well as in the initial state are taken into account microscopically. Our results are compared with recent data from the COSY and CELSIUS accelerator facilities. Reasonable agreement with available near-threshold cross section data for the reactions $p p \rightarrow p p \eta, p n \rightarrow p n \eta$, and $p n \rightarrow d \eta$ is found.
\end{abstract}

PACS: 25.10.+s, 13.75.-n, 25.40.-h

FZJ-IKP-TH-2002-16 


\section{INTRODUCTION}

Meson production in nucleon-nucleon $(N N)$ and nucleon-nucleus collisions is a rather attractive research field in hadron physics at intermediate energies. The revived interest in such reactions is closely connected to recent successful developments in the accelerator technology which enabled experimentalists to perform rather accurate measurements on the production of $\pi, \eta, \eta^{\prime}$ and other mesons in nucleon-nucleon collisions in the near-threshold region [1-3]. These data in combination with the theoretical analysis open the unique possibility to study the mechanism of the production of different mesons as well as to produce information on resonance properties, baryon-baryon and meson-baryon interactions, short range properties of the $N N$ interaction etc..

Among those reactions the production of the $\eta$ meson is certainly rather interesting. E.g., the $\eta$ is the only other non-strange pseudo Goldstone boson existing and thus a close though heavier relative of the pion. Also it is expected that $\eta$ production near threshold is closely linked with the properties of the $S_{11} N^{*}(1535)$ resonance. Finally, and may be most importantly there is already a wealth of rather accurate data on $\eta$ meson production near threshold providing a sensible testing ground for model calculations. Apart from total cross sections for the reaction $p p \rightarrow p p \eta[4-9]$, there are also some data on the total cross sections of the reactions $p n \rightarrow p n \eta[10]$ and $p n \rightarrow d \eta[11,12]$. Furthermore, there are data on differential cross sections and invariant-mass spectra $[9,13-15]$ and even anlyzing powers for the reaction $p p \rightarrow p p \eta[16]$.

The present work is devoted to the study of the production of $\eta$ mesons in $N N$ collisions. The goal is a combined theoretical analysis of all measured channels of the reaction $N N \rightarrow$ $N N \eta$, namely $p p \rightarrow p p \eta, p n \rightarrow p n \eta$ and $p n \rightarrow d \eta$, by taking into account consistently the interaction between the nucleons in the final as well as in the initial state and by utilizing a microscopic model of meson-nucleon $(M N)$ scattering $[17,18]$ for the description of the $\eta$-meson production process. We focus on the description of $\eta$-meson production in the near-threshold region, i.e. for excess energies $Q$ up to around $50 \mathrm{MeV}$. Therefore, we will restrict our investigation to $\mathrm{S}$-wave contributions. Recent data on differential cross sections of the reaction $p p \rightarrow p p \eta[14]$ suggest that in this energy region the production of $\eta$ mesons still takes place mainly in S-waves.

In spite of the abundance of theoretical investigations on $\eta$-meson production [19-32] the leading production mechanism is still not identified. There is general consent in the literature that $\eta$-meson production is dominated by re-scattering processes as depicted in Fig. 1a, with a variety of intermediate meson exchanges. However, it is not clear which one of the possible meson exchanges plays the most important role. For instance, in Refs. [21,22,25,26] it is suggested that the dominant contributions should come from the $\rho$ meson, whereas in $[27,30]$ it is found to be of minor importance compared to other contributions and in [24] that particular contribution is not even considered. Recently Nakayama et al. [32] compared the two scenarios of a $\pi$ - and a $\rho$-exchange dominance and concluded that both these scenarios can describe the cross section data for the various $N N \rightarrow N N \eta$ channels equally well.

Apart from the choice of the exchanged mesons there are also differences in the construction of the $M N \rightarrow \eta N$ transition amplitude. Many of the models [19-22,25,27,32] are based on the calculation of tree level re-scattering diagrams where the $M N \rightarrow \eta N$ transition am- 
plitude is simply parametrized by the $S_{11} N^{*}(1535)$ resonance. Background contributions, e.g., through $t$-channel meson exchanges are either omitted or simply added on top of the resonance contribution. In such approaches the relative phases between different meson exchanges have to be fixed by hand, which naturally introduces large uncertainties. As a consequence quite contradictory prescriptions have been adopted in the literature. E.g., in Ref. [21] it was tried to minimize interferences between the various production mechanisms. Assuming that the $\eta$ 's are produced only via $N^{*}$ excitation led to $\pi$ and $\rho$ exchange contributions that are orthogonal to each other and, therefore, don't interfere at all. On the other hand, in a recent investigation by Fäldt and Wilkin [26] interferences are sort of maximized. Those authors argue that only a strong interference of the $\rho$ exchange amplitude with the $\pi$ exchange contribution allows to achieve a consistent description of the experimentally observed cross sections for the reactions $p p \rightarrow p p \eta$ and $p n \rightarrow p n \eta$.

In the present investigation we employ $M N \rightarrow \eta N$ amplitudes generated from a microscopic (meson-exchange) model of the $\pi N$ system. It is a coupled-channels model that contains 5 channels, namely $\pi N, \eta N, \rho N, \sigma N$, and $\pi \Delta$ [18]. The interactions in and between the various channels are derived in the meson-exchange picture starting out from effective chiral Lagrangians. The model includes the $N^{*}(1535)$ resonance as essential contribution but in addition also various ( $t$-channel) meson and ( $u$-channel) baryon exchange diagrams. The parameters of the model are fixed by requiring a simultaneous description of the $\pi N$ phase shifts and inelasticities as well as of the transition cross sections for the reactions $\pi N \rightarrow \eta N$ and $\pi N \rightarrow \rho N$ over an energy range that extends well beyond the $\eta N$ threshold. The reaction amplitudes are obtained from solving a coupled-channels relativistic scattering equation of Lippmann-Schwinger type. Clearly, in such a model not only the $\pi N \rightarrow \eta N$ amplitude is determined by empirical data, but also the transition amplitudes involving heavier mesons are to a large extend constrained by the phase shifts and inelasticity parameters of $\pi N$ scattering. This is also true for the relative phases between the various amplitudes.

Another uncertainty in investigations of the reaction $N N \rightarrow N N \eta$ is caused by the treatment of the $N N$ FSI and ISI effects. In many papers concerned with meson production FSI effects are taken into account approximately by applying methods which are rather questionable if one wants to obtain absolute predictions for the production cross sections (see, e.g., Refs. [19-21,23,27] for the case of $\eta$-production), as was pointed out recently in Refs. [33,34]. The situation with the ISI is even less satisfying. Indeed, its effect is simply omitted in most studies.

In our investigation we take the FSI and ISI in the $N N$ system fully into account. In particular, we employ an $N N$ model that reproduces the relevant $N N$ phase shifts reasonably well up to energies around the $\eta$ production threshold and, therefore, allows a consistent description of the FSI and ISI effects.

Let us mention here that, at the present stage, the Jülich $M N$ model does not include the $\omega N$ channel. Thus, we cannot take into account the $\omega$-rescattering contribution to the reaction $N N \rightarrow N N \eta$. Anyhow, we want to emphasize that most model studies indicate that its contribution should be small $[20,21,26,32]$.

The paper is structured in the following way: In Sect. II we describe the ingredients that enter into our model calculation of $\eta$ production in nucleon-nucleon collisions. In particular, we present some details about the meson-nucleon amplitudes that are utilized for constructing 
the elementary $\eta$ meson production operator and we specify the relevant vertex parameters. We also provide a brief description of the $N N$ model that is used for the treatment of the ISI and FSI, and we supply its prediction for those energies and partial waves in the initial $N N$ state, relevant for $\eta$ production near threshold. Our results are presented and discussed in Sect. III. We show our predictions for various charge channels, i.e. $p p \rightarrow p p \eta, p n \rightarrow p n \eta$, and $p n \rightarrow d \eta$, and compare them with available data on total cross sections. We also study the sensitivity of our results to variations in the elementary $(\rho N \rightarrow \eta N)$ production amplitude and to differences in the $N N$ interaction in the final state. Finally, we analyse the role played by the various production mechanisms included in our model. A summary and some concluding remarks are given in Sect. IV.

\section{MODEL CALCULATION OF THE REACTION $N N \rightarrow N N \eta$}

We study $\eta$-meson production in distorted wave Born approximation (DWBA). This means that the transition amplitude from the initial to the final state, $M_{2 \rightarrow 3}$, is given by the expression

$$
M_{2 \rightarrow 3}=\left\langle\chi_{f}\left|\left(1+T_{N N}^{F S I} G_{0}\right) A_{2 \rightarrow 3}\left(1+G_{0} T_{N N}^{I S I}\right)\right| \chi_{i}\right\rangle .
$$

where $A_{2 \rightarrow 3}$ is the (elementary) production operator and $T_{N N}^{I S I}$ and $T_{N N}^{F S I}$ are the $N N$ reaction amplitudes in the initial and final states. A graphical representation of Eq. (1) is given in Fig. 2. Note that here we have tacitly assumed that the final-state effects are dominated by the interaction in the $N N$ system and that the interaction in the $\eta N$ system can be neglected. There is, however, experimental evidence $[6,11,35]$ that the $\eta N$ interaction has a significant impact on the energy dependence of the cross section. Under such circumstances it would be, in principle, desirable to include the $\eta N$ FSI consistently and to solve 3-body integral equation of Faddeev type for the most difficult case when all particles are in the continuum (see, e.g. Ref. [36] and references therein). A few efforts along this line can be already found in the literature [37,38], though only for the quasi two-body reaction $n p \rightarrow d \eta$. On the other hand, the experiments also indicate that the $\eta N$ interaction might have an influence only for very small excess energies, i.e. up to around 10-15 MeV above the $\eta$ threshold. Thus, a sensible investigation of the $\eta$-meson production mechanisms should be still feasible without taking into account the $\eta N$ interaction, if one focusses on the energy range $10 \leq Q \leq 50 \mathrm{MeV}$, say. In any case it is quite reasonable to consider the much more complicated 3-body effects only after a detailed quantitative understanding of $N N$ ISI and FSI effects as well as of the production process has been achieved. Therefore, in this work we disregard possible effects from the $\eta N$ FSI.

The production amplitude $A_{2 \rightarrow 3}$ in our model consists of the re-scattering diagrams with $\pi, \rho, \eta, \sigma$ meson exchanges and the direct $\eta$ production. Corresponding diagrams included in our work are shown in Fig. 1.

One of the principal novelties of our investigation is the utilization of a realistic microscopic model for the elementary reaction amplitudes $M N \rightarrow \eta N$, namely a coupled-channels model for $\pi N$ scattering that has been developed recently by the Jülich group [18,39]. The important aspect connected with the use of this model is that the off-shell properties of those 
amplitudes and also the relative phases between different meson contributions are now fixed and (to a certain degree) constrained by the data on $\pi N$ scattering. Another merit of our model study of $\eta$ production in $N N$ collisions consists in the full and consistent treatment of effects from the $N N$ FSI as well as ISI.

As mentioned already we restrict our investigation to the S-wave contributions. Recently measured angular distributions in the reaction $p p \rightarrow p p \eta$ exhibit an isotropic structure at the excess energies $Q=15 \mathrm{MeV}$ as well as at $Q=41 \mathrm{MeV}$ [14], thus suggesting that even up to $Q \approx 50 \mathrm{MeV}$ the production of $\eta$ mesons might take place predominantly in S-waves. We want to point out, however, that the invariant-mass distributions reported in the same paper cannot be described by S-waves plus $p p$ FSI alone [14]. This could be an evidence that at least at the higher energy $\mathrm{P}$-waves already play a role [40].

Restricting ourselves to S-waves we have only two amplitudes corresponding to the possible final states ${ }^{1} S_{0} s$ (for isospin $\mathrm{I}=1$ ) and ${ }^{3} S_{1} s$ (for isospin $\mathrm{I}=0$ ). The corresponding initial $N N$ states are ${ }^{3} P_{0}$ and ${ }^{1} P_{1}$, respectively. (We use the standard nomenclature where capital letters denote the $N N$ partial waves and the small letter labels the orbital angular momentum of the $\eta$ with respect to the $N N$ system.)

\section{A. Model of the meson-nucleon $(M N)$ interaction}

In this subsection we describe the structure and results of the $M N$ model - which is one of the main ingredients of our calculation. This model was developed in Refs. $[17,18,39]$. The ambitious aim of the model is a simultaneous description of the $\pi N$ phase shifts and inelasticities as well as of the transition cross sections for the reactions $\pi N \rightarrow \eta N$ and $\pi N \rightarrow \rho N$. The reaction amplitudes are obtained from solving a relativistic coupled-channels scattering equation of Lippmann-Schwinger type. The model includes 5 channels, namely $\pi N$, $\eta N, \rho N, \sigma N$, and $\pi \Delta$. All the $M N$ interactions and transition potentials are constructed in time-ordered perturbation theory (TOPT) on the basis of effective chiral Lagrangians. Most of the coupling constants at the meson-baryon-baryon and meson-meson-meson vertices included in the model are taken from other sources [18]. The cutoff masses in the vertex form factors are the only free parameters which were determined by a fit to the $\pi N$ partial-wave amplitudes. The potentials consist of $t$-channel meson exchanges, $s$-channel resonance graphs and $u$-channel baryon exchanges (cf. Figs. 2-5 in Ref. [18]).

The model provides a satisfactory overall description of all $\pi N$ partial amplitudes with total angular momentum $J \leq 3 / 2$ [18]. Specifically, in the $S_{11}$ partial wave, which is the relevant one for the reaction $N N \rightarrow N N \eta$ close to threshold, the agreement with the experimental information in a region of about $60 \mathrm{MeV}$ above the $\eta$ threshold (the $\eta N$ threshold is at $E_{c m} \approx 1486 \mathrm{MeV}$ ) is quite good, as demonstrated in Fig. 3 by the dashed lines. Therefore this model is a reasonable starting point for our calculation. The offshell transition amplitudes of this model are utilized for the meson rescattering amplitudes $M N \rightarrow \eta N$ that enter into the evaluation of the production operator $A_{2 \rightarrow 3}$ (cf. the filled circle in Fig. 1a).

It should be said, however, that the data on $\pi N$ scattering (plus the $\pi N \rightarrow \eta N$ and $\pi N \rightarrow \rho N$ transition cross sections) do not fully determine all the transition potentials 
of the coupled-channels model. E.g., in the original model [18] no direct $\rho N \rightarrow \eta N$ transition potential at all was included, because the $\pi N$ results are not too sensitive to such contributions and, accordingly, corresponding parameters could not be fixed unambigously. Possible contributions to $\rho N \rightarrow \eta N$ can arise from $t$-channel meson-exchange (e.g., $\rho, b_{1}$, etc.), from $N$ exchange and, in particular, from the $s$-channel pole diagram involving the $S_{11} N^{*}(1535)$ resonance, as shown in Fig. 4.

Note that the original $\pi N$ model [18] still generates a $\rho N \rightarrow \eta N$ transition amplitude - even in the absense of a direct $\rho N \rightarrow \eta N$ transition potential. Such an amplitude arises naturally in a coupled-channels model, e.g. via higher order transitions of the kind $\rho N \rightarrow \pi N \rightarrow \eta N$.

In view of the importance of the $\rho N \rightarrow \eta N$ transition amplitude in many of the earlier investigations of $\eta$ production, cf. the Introduction, and specifically in view of the crucial role it plays in the analysis of Fäldt and Wilkin [26] we want to explore how well this amplitude is constrained within our coupled-channels model and to which extend it can be varied. For this purpose we have created a variant of the $M N$ model where we now explicitly include a coupling of the $\rho N$ channel to the $S_{11} N^{*}(1535)$ resonance, cf. the diagrams in Fig. 4. Evidently, we need to make sure that the description of the $\pi N$ data in the region not far from the $\eta$ threshold remains basically unchanged when those diagrams are added to the model. The bare coupling constants $g_{\pi N N^{*}}, g_{\eta N N^{*}}$, and $g_{\rho N N^{*}}$ have been varied to explore the variation in the amplitude for $\rho N \rightarrow \eta N$ while staying as close as possible to the experimental phase shift and inelasticity of the $S_{11} \pi N$ partial wave (cf. Fig. 3) and the $\pi N \rightarrow \eta N$ transition cross section produced by the original model (cf. Fig. 5) in a region of about $60 \mathrm{MeV}$ around the $\eta N$ threshold.

All other $\pi N$ partial waves remain unchanged since the parameters of the t- and u-channel exchange potentials were not varied.

Eventually we settled on a small and negative value for the bare coupling constant $g_{\rho N N^{*}}$ since it turned out that only choosing it to be negative allows to achieve a significant influence of those additional diagrams on the $\rho N \rightarrow \eta N$ amplitude and, in turn, on the predictions for $\eta$ production in $N N \rightarrow N N \eta$ reactions. In Sec. III we present results for this extended model together with those based on the original $M N$ model.

As is evident from Fig. 5 the present $M N$ model of the Jülich group overestimates the $\pi^{-} p \rightarrow \eta n$ cross section by roughly $15 \%$. In order to estimate the impact of this shortcoming on the results for $N N \rightarrow N N \eta$ we performed some exploratory calculations based on a reduced $\pi N \rightarrow \eta N$ amplitude. Specifically, we weakened the $\pi N \rightarrow \eta N$ transition potential of the $M N$ model [18] phenomenologically by decreasing the coupling of the $a_{0}$ meson at the $a_{0} \pi \eta$ vertex [42] so that the transition cross section is reproduced. (Note that the $\pi N$ phase shifts are no longer described in this case!) It turned out that a decrease in $\sigma_{\pi^{-} p \rightarrow \eta n}$ by $15 \%$ implies a reduction in the predictions for $\sigma_{N N \rightarrow N N \eta}$ by roughly the same amount.

\section{B. Vertex parameters}

Besides the $M N \rightarrow \eta N$ amplitude the production amplitude $A_{2 \rightarrow 3}$ also contains the mesonnucleon-nucleon $(M N N)$ vertices from where the rescattered mesons are emitted, cf. Fig. 1a. In this subsection we want to provide the parameters involved in those vertices, i.e. 
the coupling constants and the cutoff masses in the form factors. As far as the coupling constants are concerned all of them are taken over from the full Bonn $N N$ model [43]. The only exception is the one of the $\eta$ meson - because this meson is not included in the full Bonn model. Here we take the value which follows from $\mathrm{SU}(6)$ symmetry, i.e. $g_{\eta N N}^{2} /(4 \pi)=1.8$, which is also used in the $M N$ model [18] for the $\eta N N$ vertex in the nucleon-exchange diagrams.

The vertex form factors are likewise taken over from the full Bonn $N N$ model or (in case of the $\eta$ meson) from the $\pi N$ model [18]. In those models the form factors are assumed to be of conventional monopole form, i.e. $F\left(\vec{q}_{M}\right)=\left(\Lambda_{M N N}^{2}-m_{M}^{2}\right) /\left(\Lambda_{M N N}^{2}+\vec{q}_{M}^{2}\right)$, where $\Lambda_{M N N}$ is the cutoff parameter and $\vec{q}_{M}$ and $m_{M}$ are the 3-momentum and mass of the exchanged meson, respectively. The values of the employed vertex parameters are summarized in Table I.

Since the elementary $M N \rightarrow \eta N$ amplitude and also the vertex parameters are fixed from earlier investigations we do not have any free and/or adjustable parameters in the production amplitude $A_{2 \rightarrow 3}$. Thus, our results for the reaction $N N \rightarrow N N \eta$ are genuine model predictions.

\section{C. $N N$ interaction in the initial state}

The laboratory energy corresponding to the $\eta$-production threshold is $T_{l a b}=1250 \mathrm{MeV}$. Thus, the $N N$ interaction in the initial state takes place at rather high energies. At such energies the effects of the ISI are characterized by the following features:

- The ISI has practically no influence on the energy dependence of the $\eta$ production cross section because the variation of the $N N$ interaction in the initial state within the energy interval determining the threshold region is negligible [44].

- The $N N$ scattering in the energy region in question is already strongly inelastic because multiple-pion production channels are open. In Ref. [33] it is shown that in such a case the ISI leads to a significant reduction of the cross section.

Indeed, the energy relevant for the ISI is so high that the well known realistic $N N$ models such as the Bonn and the Paris potentials [43,45] can not be applied anymore. In case of the Paris or the Bonn one-boson-exchange (OBE) potentials no inelastic channels were included and therefore they are not valid for energies above $\pi$ production threshold. In the full Bonn model such inelastic channels are, in principle, build in via couplings to the $N \Delta$ and $\Delta \Delta$ channels. However, since meson retardation effects are retained as well three body singularities occur for energies above $\pi$ production threshold and hence the calculation is technically much more difficult [46]. Therefore, in the following we use an alternative $N N$ model, CCF [47], which contains the same dynamics as the full Bonn model, specifically the coupling to $N \Delta$ and $\Delta \Delta$ channels, but where meson retardation effects are removed by the so-called folded diagrams technique. In Figs. 6 and 7 we present the results of the CCF model [47] for the $N N$ phase shifts and inelasticities in the ${ }^{3} P_{0}$ and ${ }^{1} P_{1}$ partial waves. Since we restrict ourselves to final states composed purely out of $s$-waves, these are the relevant partial waves for the $N N$ interaction in the inital state. The agreement with the 
experimental data is quite good, specifically in view of the fact that the model was only fitted to the $N N$ phase shifts below the pion production threshold, i.e. for $T_{l a b} \leq 300 \mathrm{MeV}$. Note, that in this model the inelasticities are introduced only through the coupling to channels involving the $\Delta$ isobar. Therefore, in the ${ }^{1} P_{1}$ channel, where only the simultaneous exitation of two $\Delta$ isobars is allowed, the inelasticity becomes noticable only after $600 \mathrm{MeV}$ kinetic energy, i.e. somewhat later than indicated by the empirical data. However, in the energy range relevant for $\eta$ production the description is reasonable. Finally, let us mention that the width of the $\Delta$ isobar is taken into account phenomenologically by using a complex $\Delta$ mass in the propagator. Specifically, we employ a parametrization of the width given by Kloet and Tjon in Ref. [49] which is energy- as well as momentum-dependent.

The evaluation of the ISI effects amounts to performing a loop integration, cf. Eq. (1) and Fig. 2, which gives rise to contributions from the unitarity cut and from the principal value (PV) integral. Since in the initial state the colliding nucleons have rather large relative momenta it was argued in Ref. [33] that the contribution of the PV integral should be suppressed as compared to the piece resulting from the on-shell (unitarity) cut. Under this assumption the ISI effect on the cross section reduces to a simple multiplicative factor $|\lambda|^{2}$ which can be expressed in terms of on-shell $N N$ information only, namely phase shifts $(\delta)$ and inelasticities $(\eta)$ :

$$
|\lambda|^{2}=\left|1-i \frac{\pi E_{p}}{2} p T_{N N}\right|^{2}=\frac{1}{4}|1+\eta \cos (2 \delta)+i \eta \sin (2 \delta)|^{2} .
$$

Here, the T-matrix is defined as $T=-\frac{2}{\pi E_{p}} \frac{\eta e^{2 i \delta}-1}{2 i p}$ and $p\left(E_{p}\right)$ is the cms momentum (energy) of the nucleons.

In our actual calculations it turns out that there is a strong cancellation between the Born term and the piece coming from the unitarity cut, i.e. between the terms corresponding to the "1" and to " $\eta \cos (2 \delta)$ " in Eq. (2). Then the ISI factor $\lambda$ in Eq. (2) is rather small. But in such a case, the contribution from the PV integral, which is certainly small and which was neglected in Eq. (2), becomes important as well and has an influence on the ISI effects. We will come back to this point in the next section.

Due to 3-body singularities appearing in the loop with the ISI the evaluation of the PV integral is very involved [50]. In the present work we avoid the technically rather tedious explicit treatment of this 3-body singularity. Rather we follow the approach which was already used in the work of Batinic et al. [24] and suppress the 3-body singularity by putting the nucleon energies appearing in the meson propagator in the intermediate state on the energy shell. We want to emphasize that this approximation still provides the correct value for the contribution from the two body $N N$ unitarity cut in the ISI loop. Only the contribution from the PV integral is influenced by the approximation discussed above.

The effects of the ISI on the results for $\eta$ production in $N N$ collisions will be discussed in detail in section III. Here we want to emphasize only that the PV integral in the ISI loop introduces an interesting feature. It breaks the universality of the ISI as it is suggested by the prescription given in Eq. (2), i.e. it implies that the reduction caused by the ISI will differ for the various contributions to $\eta$-meson production and, thus, depends also on the production mechanism. The approximative treatment of ISI according to Eq. (2) is unable to account for such effects. 


\section{D. $N N$ interaction in the final state}

Effects of the $N N$ interaction in the final state in various production reactions were investigated in detail in Refs. [33,34,51-53]. In particular, in Ref. [34] it was demonstrated that the $N N$ FSI cannot be factorized from the production amplitude if one wants to obtain reliable quantitative predictions for the cross sections. This conclusion confirms the arguments given in Ref. [33]. Also it was shown in Ref. [34] that the use of the Jost function of some realistic $N N$ potentials for the evaluation of FSI effects is invalid and may lead to results considerably different from the ones based on a proper calculation. In this work $N N$ FSI effects are treated consistently, i.e. the calculation of loop integrals is performed with taking into account the full dynamics both from the $N N$ T-matrix and from the production amplitude (cf. Eq. (1) or Fig. (2), respectively). In the calculation we utilize not only the CCF [47] $N N$ model, i.e. the one which is used for the ISI as well, but also Bonn B [54] in order to examine the sensitivity of the results to the $N N$ interaction model. Both models describe equally well the $N N$ phase shifts in the ${ }^{1} S_{0}$ and ${ }^{3} S_{1}$ partial waves, which are relevant for the $\eta$ production, for energies below the pion production threshold, i.e. in the energy region relevant for the FSI.

Note that the Coulomb interaction in the $p p$ final state is taking into account, applying the prescription described in Ref. [55].

\section{RESULTS OF THE CALCULATION OF THE REACTION $N N \rightarrow N N \eta$}

Our results for the reactions $p p \rightarrow p p \eta, p n \rightarrow p n \eta$ and $p n \rightarrow d \eta$ are presented in Fig. 8 and compared with empirical information from the Uppsala/Celsius $[6,10-12]$ and Jülich/COSY $[8,9]$ accelerator facilities. The dashed curves correspond to the calculation based on the original $M N$ model (cf. section II A) for the elementary $\eta$-production amplitude and the CCF $N N$ model [47] for the ISI and FSI.

Evidently, the calculation based on the original Jülich $M N$ model [18] yields a qualitative overall description of the experimental data. This has to be certainly considered as success because, as mentioned before, there are no adjustible parameters in our calculation of $N N \rightarrow$ $N N \eta$. As one can observe from Fig. 8, for the $p p \eta$ case we overestimate the cross section by approximately $30 \%$ whereas for $p n \rightarrow p n \eta$ the model calculation underestimates the experimental data by about $50 \%$. The situation for the reaction $p n \rightarrow d \eta$ is very similar to $p n \rightarrow p n \eta$ since both processes are governed by the $I=0$ isospin channel. As is known from earlier investigations $[26,32]$ for the latter reaction the contribution of the $I=1$ channel is much smaller than the one for $I=0$.

However, it is interesting to investigate the sensitivity of our results to the $M N$ amplitude and, specifically, to see whether the model predictions for $N N \rightarrow N N \eta$ can be improved by modifications in the $M N$ model via introducing some additional graphs, as described in section II A. For the original $M N$ model the amplitudes of the $\pi$-and $\rho$-exchange contributions to $\eta$ production turned out to be almost orthogonal to each other. Thus, there is practically

no interference between these contributions. The additional diagrams which we introduced into the $M N$ model allow a slight modification of the orientation of these amplitudes in the 
complex plane and, as a consequence, now interference effects do occur. Specifically, it is possible to generate a destructive interference in the isotriplet channel $(I=1)$ and a constructive interference in the $I=0$ case. This leads to a slight reduction of the cross section in the reaction $p p \rightarrow p p \eta$ and to a significant enhancement for $p n \rightarrow p n \eta$, cf. the solid lines in Figs. 8, bringing now the results quite close to the experiment. Also the prediction for the reaction $p n \rightarrow d \eta$ is now in good agreement with the experimental data.

\section{A. Contributions of individual meson exchanges.}

Let us examine our model in more detail and analyze the contributions of the individual exchange diagrams. We do this for the calculation based on the extended $M N$ model. The main differences between the results obtained for the original $M N$ model and its extended version will be discussed at the end of this section.

Corresponding results are presented in Fig. $9(p p \rightarrow p p \eta)$ and in Fig. $10(p n \rightarrow p n \eta$ for $I=0)$, where we show the production cross section for the individual meson exchanges together with the full results. Furthermore, in Table II we compare cross sections of the full calculation with results obtained without ISI, i.e. where $T_{N N}^{I S I}$ in Eq. (1) was set to zero, at the specific excess energy $Q=35 \mathrm{MeV}$. The latter allows us not only to expose the relevance of the various meson exchange (rescattering) contributions for the $\eta$ production cross section but also to elucidate that their relative importance is strongly influenced by the ISI. For example, in the model calculation without ISI the dominant contributions come from $\pi$ and $\rho$ exchanges, both being of comparable magnitude. However, the ISI reduces the $\rho$-exchange contribution much more strongly then the one from $\pi$ exchange (cf. Table. II) leaving $\pi$ exchange as the only dominant production mechanism. The contributions from the other production mechanisms ( $\sigma$ exchange, $\eta$ exchange, etc.) are already comparably small before including the ISI and they are also significantly reduced (though not as much as the $\rho$ ) by introducing the ISI.

Let us emphasize in this context that phenomenological treatments like those based on Eq. (2) are unable to account for such effects resulting from details of the dynamics. They lead only to an overall reduction of the cross section independent of the production mechanisms, as is indicated by the last 3 columns of Table II. (Note that the reduction factors used in Ref. [32] differ from those for CCF because the ones in the former work are based on the $N N$ phase shifts listed at the SAID library [44]. The reduction factors of Ref. [26] are not obtained from Eq. (2) but from a different prescription.)

We also want to mention that the ISI effects seen in our investigation are at variance with those reported in the only other study of $\eta$-meson production where the ISI was taken into account explicitly [24]. In that work by Batinić et al. it was found that the combined distortions from the FSI and ISI are not sensitive to the dynamics of the production operator. We don't have an explanation readily at hand for this discrepancy. We can only conjecture that it might be due to differences in the employed $M N \rightarrow \eta N$ transition amplitudes.

As we have discussed above, after inclusion of the ISI $\pi$ exchange plays the dominant role in our calculation of the reaction $p p \rightarrow p p \eta$ (see Figure 9). Nevertheless, the contributions of the other mesons, especially of the $\eta$ and $\rho$ exchanges and of the direct term are still significant 
due to interference effects with the amplitude of the $\pi$ exchange. The $\eta$ exchange and the direct term contribute constructively to the reaction $p p \rightarrow p p \eta$ whereas the interferences from the $\sigma$ and $\rho$ mesons are destructive.

Note that the actual effect of those interferences depends also on whether the ISI is included or not. That can be best seen by the fact that the ISI reduces the individual meson-exchange contributions by factor 4 or more whereas the total cross section is only reduced by roughly a factor 2, cf. Table II.

Let us now come to the reaction $p n \rightarrow p n \eta$. The cross section of this reaction is primarily determined by the isovector particles $(\pi$ and $\rho)$ in the $I=0$ channel, because their contributions are weighted by the large isospin coefficient (9 versus 1 for $I=1$ ). In addition, for the relevant $N N$ partial wave in the initial state, the ${ }^{1} P_{1}$, it turned out that the variation of ISI effects for the various production mechanisms is not as pronounced as in the $I=1$ $\left({ }^{3} P_{0}\right)$ case. Indeed, except for pion exchange, in this channel the reduction due to the ISI is almost universal as can be seen in Table II.

The relative contributions of the individual meson exchanges to the reaction $p n \rightarrow p n \eta$ (in the $I=0$ channel) are shown in Fig. 10 for the full calculation. As mentioned already, the dominant role belongs again to the pion exchange, though the contribution from $\rho$ exchange is now much less suppressed as in the $I=1$ case (cf. Fig. 9). The individual contributions from the other production mechanisms are again much smaller. Like in case of $p p \rightarrow p p \eta$ interferences play a role once we add all contributions coherently. Specifically, the $\eta$ - and the $\rho$-meson exchange exhibit opposite features as in the $p p \rightarrow p p \eta$ case, i.e. the $\eta$ is destructive while the $\rho$ is constructive. The direct term acts again constructively and its contribution nearly cancels with the one resulting from $\eta$ exchange. The contribution from $\sigma$ exchange turns out to be negligible.

The interference effects between $\pi$ and $\rho$ meson exchange amplitudes for the original and extended $M N$ models are illustrated in Table III, where we present the ratio of the coherent sum of the two amplitudes to the incoherent sum. For the original $M N$ model this ratio is close to 1 (for both isospin channels) verifying that there are only small interference effects. For the extended $M N$ model those interferences are much more pronounced.

Table II lists also the phenomenological reduction factor that follows from Eq. (2) for the employed $N N$ initial state interaction (in the column with the header ' $\mathrm{CCF}$ '). Thus, we can compare directly the reduction of the cross section that follows from the explicit inclusion of the ISI with the one suggested by the phenomenological prescription. It is evident that the prescription Eq. (2) doesn't work that well for the $\pi$ and $\rho$ exchange contributions. For the $\eta$ and specifically for the $\sigma$ exchange, however, the results are fairly similar.

\section{B. Role of the $\eta N N$ coupling constant}

Now we would like to comment on the influence of the $\eta N N$ coupling constant. As mentioned before, in our calculation we take the value $g_{\eta N N}^{2} /(4 \pi)=1.8$ that follows from $\mathrm{SU}(6)$ symmetry. This value is already much smaller than those used in OBE versions of the Bonn $N N$ model [43,54], say, which are around 3 to 7 . On the other hand, some studies of $\eta$ production in $N N$ collisions suggest that only still smaller $\eta N N$ coupling constants allow 
to describe the experimental data [26,30]. E.g., a value of only about $g_{\eta N N}^{2} /(4 \pi)=0.4$ was employed in [30] while in [26] the $\eta$ contribution was even completely neglected. Therefore, it is interesting to see whether our results on $\eta$ production based on the original $M N$ model could be also improved by simply varying the $\eta N N$ coupling constant in the production operator.

In our model calculations $g_{\eta N N}$ enters via the $\eta$ exchange contribution but also in the direct term. The latter is not negligible and interferes constructively with the dominant $\pi$ exchange contribution in both isospin channels of the reaction $N N \rightarrow N N \eta$. On the other hand, as mentioned before, the $\eta$ exchange contribution interferes constructively for the reaction $p p \rightarrow p p \eta$ but destructively for the $I=0$ part of $p n \rightarrow p n \eta$. Indeed, the direct term and the $\eta$ exchange basically cancel each other in the dominant isospin $I=0$ channel of the reaction $p n \rightarrow p n \eta$ and, therefore, the $\eta N N$ coupling constant influences primarily the cross section of the $p p \rightarrow p p \eta$ reaction. Thus a reduction of $g_{\eta N N}$ would only decrease the cross section for $p p \rightarrow p p \eta$. It would not alter the results for $p n \rightarrow p n \eta$ and $p n \rightarrow d \eta$ and, therefore, does not lead to an improvement for the latter two reaction channels.

\section{Sensitivity to the $N N$ interaction.}

In order to examine the sensitivity of our results to differences in the $N N$ interaction in the final state we also performed a calculation where the OBE potential Bonn B [54] was utilized. Corresponding results are presented in Fig. 11 by dashed-dotted lines. The results with the CCF $N N$ model are also shown (solid lines) in order to facilitate a comparison. As one can see from this figure, for the $p p \rightarrow p p \eta$ channel the predictions of both $N N$ models lie basically on top of each other. However, for the $p n \rightarrow p n \eta$ channel the results based on the Bonn B potential are about 20\% larger and, indeed, are practically in agreement with the experiment. At the same time the cross section for the $p n \rightarrow d \eta$ channel is also enhanced by about $20 \%$ and now slightly overshoots the data.

In any case, the differences between the results for the two considered $N N$ models are not that large. But let us emphasize here an interesting by-product of the above comparison. Obviously it is not possible to achieve a simultaneous description of all three measured $\eta$ production channels. Either we have agreement for the reactions $p p \rightarrow p p \eta$ and $p n \rightarrow d \eta$ (for $\mathrm{CCF}$ ) and then $p n \rightarrow p n \eta$ is off, or $p p \rightarrow p p \eta$ and $p n \rightarrow p n \eta$ are reproduced (Bonn $\mathrm{B})$ and then the results for $p n \rightarrow d \eta$ deviate from the data. Let us remind the reader that, close to threshold, there are only two independent amplitudes that determine those three reaction channels. The same incompatibility is also seen in the results of Fäldt and Wilkin, cf. Fig. 5 of Ref. [26], and, therefore, we believe that it is not due to the specific production mechanism employed in our study. It remains to be seen whether this discrepancy will disappear when contributions from higher partial waves are included. In any case, we want to mention that a similar incompatibility exists also for near-threshold pion production, $\mathrm{cf}$. the results in Fig. 3 of Ref. [56] and also in Ref. [57]. 


\section{Comparison with other model calculations.}

In this subsection we want to discuss qualitatively the differences between our model and other model calculations in the literature. We restrict the comparison to such calculations where also $p p$ as well as $p n$ induced $\eta$ production channels are considered, i.e. to the works of Gedalin et al. [21] and Nakayama et al. [32], where results for the reactions $p p \rightarrow p p \eta$ and $p n \rightarrow p n \eta$ are presented, and to Ref. [26], where in addition the channel $p n \rightarrow d \eta$ is considered.

The relatively large value of 6.5 for the cross section ratio $\sigma_{p n \rightarrow p n \eta} / \sigma_{p p \rightarrow p p \eta}$ established in the CELSIUS experiment [10] revealed that the dominant $\eta$-production mechanism has to be of isovector nature. (Exchange of scalar mesons would yield a ratio of roughly one!) Furthermore, simple estimations [10,26], taking into account the different strengths in the final ( $p p$ versus $p n$ ) state interactions, strongly suggest that a production operator involving only a single isovector-meson exchange $(\pi$ or $\rho)$ might still fall short of describing the experiments and, thus, further production mechanisms should be relevant. Indeed, a scenario with a dominant contribution from isovector meson exchange is realized in all the models cited above, including ours. However, while $\rho$ exchange is the dominant $\eta$ production mechanism in Refs. [21,26] and in the alternative model of Ref. [32] it is $\pi$ exchange that provides the bulk of the cross section in our model and in the regular model of Nakayama et al. [32]. We should mention, however, that in our model $\pi$ and $\rho$ exchange are of pretty much the same strength and provide comparable cross sections before the ISI is included, as can be seen from Table II. If we would ignore ISI effects altogether, as done in Ref. [21], or use the phenomological prescription employed in Refs. [26,32] this feature would persist.

The models differ also significantly when it comes to the role played by the smaller contributions. For example in the model of Fäldt and Wilkin the $\pi$ exchange is very important. Its strong interference with the dominant $\rho$ exchange contribution is the main mechanism which allows those authors to achieve a consistent description of the experimentally observed cross sections for the reactions $p p \rightarrow p p \eta$ and $p n \rightarrow p n \eta$. On the other hand, in the alternative model of Nakayama [32] it is the interference of the $\omega$ exchange with the dominant $\rho$ exchange that is responsible for obtaining a reproduction of the data. Finally, in the regular model of the latter publication agreement with the data is achieved by the interference of the $\eta$ exchange with the dominant $\pi$ exchange.

In our model calculation based on the extended $M N$ model it is the constructive interference between the $\pi$-and $\rho$-meson contributions which yields the main enhancement in the cross sections for $p n \rightarrow p n \eta$ and $p n \rightarrow d \eta$ and brings the results close to the experiment. Thus, for the $I=0$ dominated reactions the mechanism is similar to the one in the model of Fäldt and Wilkin. However, this is no longer true for the reaction $p p \rightarrow p p \eta$. Here, in our model the $\pi-\rho$ interference does not play an important role. The reason for that are the strong effects from the ISI, discussed above, which reduce the $\rho$-exchange contributions much more strongly than those coming from $\pi$ exchange, cf. Table II and, therefore, suppress also the interference. The reproduction of the data for the reaction $p p \rightarrow p p \eta$ is mainly due to a constructive interference of the dominant $\pi$-exchange contribution with the contributions from $\eta$ exchange and from direct $\eta$ production. Thus, for the $I=1$ case the mechanism is similar to the one in the regular model of Nakayama [32]. 
In the work of Gedalin et al. [21] several interference patterns are studied. But, in general, their results do not agree that well with the experiments in the energy region $Q \leq 50 \mathrm{MeV}$ considered. Because of those reasons we refrain from a more detailed comparision with their results. Let us mention, however, that the most prominent feature in their model is the absence of any interferences between the contributions of vector meson exchange and pseudoscalar (or scalar) meson exchange. The orthogonality of those contributions is achieved by a peculiar choice of interaction Lagrangians for the vector mesons. However, it seems to us that those Lagrangians do not fulfil the requirement of time reversal invariance. Also, they seem to be in contradiction to the ones applied by these authors in their own earlier investigations [20].

Interestingly, also in our calculation based on the original $M N$ model there is practically no interference between the $\pi$ and $\rho$ exchange contributions, as already mentioned above. But in our model the origin of this feature is quite different. Here the orthogonality of the two amplitudes is generated by the dynamics of the coupled-channels $\pi N$ model. In particular, the Born term $\rho N \rightarrow N^{*}(1535) \rightarrow \eta N$ that is the main source of the orthogonality in the model of Gedalin et al. is not even present in the original $M N$ that we use in our study of $\eta$ meson production. Once this diagram is included in the coupled-channels $M N$ model (cf. the extended $M N$ model described in subsect. II A) interference effects become more pronounced rather than suppressed.

\section{SUMMARY}

We performed a detailed theoretical calculation of the different channels of the reaction $N N \rightarrow N N \eta(p p \rightarrow p p \eta, p n \rightarrow p n \eta, p n \rightarrow d \eta)$ in the near-threshold region, i.e. for excess energies up to about $50 \mathrm{MeV}$. The production mechanisms which have been included consist of re-scattering terms with $M=\pi, \rho, \eta, \sigma$ meson exchanges and the direct $\eta$ production. Effects of the final and initial state interaction between the nucleons are fully taken into account. The calculation utilizes the CCF $N N$ model for the treatment of the FSI and ISI and a realistic coupled-channels model of the $\pi N$ system for the evaluation of the $M N \rightarrow$ $\eta N$ transition amplitudes. A qualitative agreement of calculated cross sections with the experimental data is achieved for all considered $\eta$ production channels. This has to be certainly considered as success because there are no adjustable parameters in this model calculation. It is also shown that even a quantitative description of the data can be obtained if one introduces small modifications of the $M N \rightarrow \eta N$ amplitudes by exploiting some freedom in the $\rho N \rightarrow \eta N$ transition potential of the original $M N$ model.

In our model the dominant role in $\eta$-meson production near threshold belongs to the rescattering mechanism with intermediate pion exchange. The contributions from other meson exchanges, specifically from $\rho$ and $\eta$ as well as from the direct $\eta$ production are smaller. However, these mechanisms are still important and have an influence on the cross sections due to their interference with the amplitude corresponding to the $\pi$ exchange.

Our study shows that ISI as well as FSI effects are not universal but depend, among others, on the concrete meson production mechanisms. Thus, a consistent treatment of these effects is very important for a quantitative comparison of model calculations with available experimental data on the considered reactions. Specifically, the interaction in the initial 
$N N$ system plays a crucial role since it affects the magnitude of the cross sections, leading to a significant reduction.

The presented model should be viewed as a first step towards a consistent description of $\eta$ production in $N N$ collisions and in meson induced reactions. Future work should avoid some of the technical approximations, e.g. by an explicit treatment of the three-body singularities [50]. In addition, and most importantly, further mechanisms for $\eta$-meson production should be explored. As mentioned, $\eta$-meson production via $\omega$ rescattering is still missing in the present model but should be taken into account. Furthermore, rescattering contributions involving the $\Delta$ isobar in the two baryon intermediate states $[56,58]$ should be investigated. Finally, higher partial waves should be included in order to facilitate an extension of the model calculation to higher excess energies and also to make a comparison with measured angular distributions, polarizations and invariant mass spectra meaningful.

\section{ACKNOWLEDGEMENTS}

We would like to thank P. Moskal and K. Nakayama for fruitful discussions. This work was partly supported by the DFG-RFBI grant No. 02-02-04001 (436 RUS 113/652/1-1) and by the RFBR grant No. 00-15-96562. 


\section{REFERENCES}

[1] H. Machner and J. Haidenbauer, J. Phys. G25, 231 (1999).

[2] P. Moskal, M. Wolke, A. Khoukaz, and W. Oelert, hep-ph/0208002, Prog. Part. Nucl. Phys 49, 1 (2002).

[3] G. Fäldt, T. Johansson, and C. Wilkin, Phys. Scr. T99, 146 (2002).

[4] A.M. Bergdolt et al., Phys. Rev. D 48, 2969 (1993).

[5] E. Chiavassa et al., Phys. Lett. B 322, 270 (1994).

[6] H. Calén et al., Phys. Lett. B 366, 366 (1996).

[7] F. Hibou et al., Phys. Lett. B 438, 41 (1998).

[8] J. Smyrski et al., Phys. Lett. B474 , 182 (2000).

[9] P. Moskal et al., $\pi \mathrm{N}$ Newsletter 16, 367 (2002); nucl-ex/0110018.

[10] H. Calén et al., Phys. Rev. C 58, 2667 (1998).

[11] H. Calén et al., Phys. Rev. Lett. 80, 2069 (1998).

[12] H. Calén et al., Phys. Rev. Lett. 79, 2642 (1997).

[13] H. Calén et al., Phys. Lett. B 458, 190 (1999).

[14] M. Abdel-Bary et al., nucl-ex/0205016.

[15] P. Moskal et al., nucl-ex/0208004, nucl-ex/0210019.

[16] P. Winter et al., Phys. Lett. B544, 251 (2002); P. Winter, diploma thesis, Jül-3943, Forschungszentrum Jülich (2002).

[17] C. Schütz, J. Haidenbauer, J.W. Durso, and J. Speth, Phys. Rev. C 57, 1464 (1998).

[18] O. Krehl, C. Hanhart, S. Krewald, and J. Speth, Phys. Rev. C 62, 025207 (2000).

[19] J.M. Laget, F. Wellers, and J.F. Lecolley, Phys. Lett. B257, 254 (1991).

[20] A. Moalem, E. Gedalin, L. Razdolskaja, and Z. Shorer, Nucl. Phys. A600, 445 (1996); Nucl. Phys. A589, 649 (1995).

[21] E. Gedalin, A. Moalem, and L. Razdolskaja, Nucl. Phys. A 634, 368 (1998).

[22] A.B. Santra and B.K. Jain, Nucl. Phys. A 634, 309 (1998).

[23] V. Bernard, N. Kaiser, and Ulf-G. Meißner, Eur. Phys. J. A 4, 259 (1999).

[24] M. Batinić, A. Švarc, and T.-S.H. Lee, Phys. Scr. 56, 321 (1997).

[25] J.-F. Germond and C. Wilkin, Nucl. Phys. A518, 308 (1990).

[26] G. Fäldt and C. Wilkin, Phys. Scr. 64, 427 (2001).

[27] T. Vetter, A. Engel, T. Biró, and U. Mosel, Phys. Lett. B 263, 153 (1991).

[28] N.I. Kochelev, V. Vento, and A.V. Vinnikov, Phys. Lett. B 472, 247 (2000).

[29] M. Dillig, hep-ph/0202067.

[30] M.T. Peña, H. Garcilazo, and D.O. Riska, Nucl.Phys. A683, 322 (2001).

[31] V. Yu. Grishina, L.A. Kondratyuk, M. Büscher, C. Hanhart, J. Haidenbauer, and J. Speth, Phys. Lett. B475, 9 (2000).

[32] K. Nakayama, J. Speth, and T.-S. H. Lee, Phys. Rev. C 65, 045210 (2002).

[33] C. Hanhart and K. Nakayama, Phys. Lett. B454, 176 (1999).

[34] V. Baru, A.M. Gasparian, J. Haidenbauer, A.E. Kudryavtsev, and J. Speth, Phys. Atom. Nucl. 64, 579 (2001); nucl-th/0006075.

[35] P. Moskal et al., Phys. Lett. B 482, 356 (2000).

[36] E. W. Schmid and H. Ziegelmann, "The quantum mechanical three-body problem", Pergamon press, Braunschwieg, Vieweg, 1974.

[37] T. Ueda, Prog. Theor. Phys. 87, 983 (1992).

[38] H. Garcilazo and M.T. Peña, Phys. Rev. C 66, 034606 (2002). 
[39] O. Krehl, PhD thesis, Jül-3692, Forschungszentrum Jülich (1999).

[40] K. Nakayama et al., in preparation.

[41] A. Baldini, V. Flaminio, W.G. Moorhead, and D.R.O. Morrison, in: Landolt-Börnstein, Vol. 12a, edited by H. Schopper (Springer, Berlin, 1988). References to the original data can be traced back from this compilation.

[42] A. Sibirtsev, S. Schneider, Ch. Elster, J. Haidenbauer, S. Krewald, and J. Speth, Phys. Rev. C 65, 044007 (2002).

[43] R. Machleidt, K. Holinde, and Ch. Elster, Phys. Report 149, 1 (1987).

[44] CNS DAC Services, http://gwdac.phys.gwu.edu

[45] M. Lacombe, B. Loiseau, J. M. Richard, R. Vinh Mau, J. Côté, P. Pirés, and R. de Tourreil, Phys. Rev. C 21, 861 (1980).

[46] C. Elster, K. Holinde, D. Schütte, and R. Machleidt, Phys. Rev. C 38, 1828 (1988).

[47] J. Haidenbauer, K. Holinde, and M.B. Johnson, Phys. Rev. C 48, 2190 (1993).

[48] R.A. Arndt, L.D. Roper, R.A. Bryan, R.B. Clark, B.J. VerWest, and P. Signell Phys. Rev. D 28, 97 (1983).

[49] W.M. Kloet and J.A. Tjon, Phys. Rev. C 30, 1653 (1984).

[50] A. Motzke, C. Elster, and C. Hanhart, nucl-th/0207047 (2002); Phys. Rev. C, in print.

[51] K. M. Watson, Phys. Rev. 88, 1163 (1952).

[52] A. B. Migdal, Sov. Phys. JETP 1, 2 (1955).

[53] J. A. Niskanen, Phys. Lett. B 456, 107 (1999).

[54] R. Machleidt, Adv. Nucl. Phys. 19, 189 (1989).

[55] C. Hanhart, J. Haidenbauer, A. Reuber, C. Schütz and J. Speth, Phys. Lett. B 358, 21 (1995).

[56] C. Hanhart, J.Haidenbauer, O. Krehl, J. Speth, Phys. Lett. B 444, 25 (1998).

[57] G. Fäldt and C. Wilkin, Phys. Rev. C 56, 2067 (1997).

[58] C. Hanhart, J.Haidenbauer, O. Krehl, J. Speth, Phys. Rev. C 61, 064008 (2000). 


\section{TABLES}

TABLE I. Meson masses, coupling constants and cutoff masses utilized in the calculation. The values are taken from the cited references. Monopole type form factors are used at all mesons-baryon vertices.

\begin{tabular}{|c|c|c|c|c|}
\hline \hline & $m_{M}(\mathrm{MeV})$ & $\frac{g_{M N N}^{2}}{4 \pi}$ & $\Lambda_{M N N}(\mathrm{MeV})$ & Ref. \\
\hline$\pi$ & 138.03 & 14.4 & 1300 & {$[43]$} \\
$\rho$ & 769 & $0.84\left(\kappa_{\rho}=6.1\right)$ & 1400 & {$[43]$} \\
$\eta$ & 547.45 & 1.8 & 1500 & {$[18]$} \\
$\sigma$ & 550 & 5.689 & 1700 & {$[43]$} \\
\hline \hline
\end{tabular}

TABLE II. Contributions of individual meson exchanges to the $N N \rightarrow N N \eta$ cross section and the influence of the $N N$ initial state interaction. The values correspond to the excess energy $Q$ $=35 \mathrm{MeV}$. The column labelled 'ratio' exemplifies the actual reduction of the cross section after inclusion of the ISI. For comparison we also present phenomenological reduction factors employed in the model calculations of Nakayama et al. [32] and Fäldt and Wilkin [26]. In the column 'CCF' we give the phenomenological reduction factor that follows from Eq. (2) for the employed $N N$ initial state interaction.

\begin{tabular}{|c|c|c|r||r|r|r|}
\hline \hline & \multicolumn{3}{|c||}{$\sigma_{N N \rightarrow N N \eta}[\mu b]$} & \multicolumn{3}{c|}{$\begin{array}{c}\text { phenomenological } \\
\text { reduction factors }\end{array}$} \\
\hline$p p \rightarrow p p \eta$ & with ISI & without ISI & ratio & CCF & Ref. [32] & Ref. [26] \\
\hline$\pi$ & 3.11 & 9.35 & 0.33 & $\uparrow$ & $\uparrow$ & $\uparrow$ \\
$\eta$ & 0.25 & 10.3 & 0.02 & & & \\
$\sigma$ & 0.24 & 3.01 & 0.08 & 0.13 & 0.19 & 0.59 \\
full result & 0.36 & 3.17 & 0.11 & & & $\downarrow$ \\
\hline$p n \rightarrow p n \eta$ (I=0) & 5.69 & 10.4 & 0.55 & $\downarrow$ & $\downarrow$ & Ref. [26] \\
\hline$\pi$ & with ISI & without ISI & ratio & CCF & Ref. [32] \\
$\rho$ & 10.4 & 26.12 & 0.4 & $\uparrow$ & $\uparrow$ & $\uparrow$ \\
$\eta$ & 4.30 & 25.96 & 0.17 & & & \\
$\sigma$ & 0.16 & 0.85 & 0.19 & 0.30 & 0.27 & 0.53 \\
full result & 0.27 & 1.05 & 0.26 & & $\downarrow$ & $\downarrow$ \\
\hline \hline
\end{tabular}


TABLE III. Illustration of interference effects between the reaction amplitudes based on the $\pi$ and $\rho$ meson-exchange contributions. The values correspond to the excess energy $Q=35 \mathrm{MeV}$.

\begin{tabular}{|c|c|c|}
\hline \hline & Original model & Extended model \\
\hline & $\frac{\left|M_{\pi}+M_{\rho}\right|^{2}}{\left|M_{\pi}\right|^{2}+\left|M_{\rho}\right|^{2}}$ & $\frac{\left|M_{\pi}+M_{\rho}\right|^{2}}{\left|M_{\pi}\right|^{2}+\left|M_{\rho}\right|^{2}}$ \\
\hline $\mathrm{I}=1$ & 0.88 & 0.66 \\
\hline $\mathrm{I}=0$ & 1.13 & 1.45 \\
\hline \hline
\end{tabular}




\section{FIGURES}

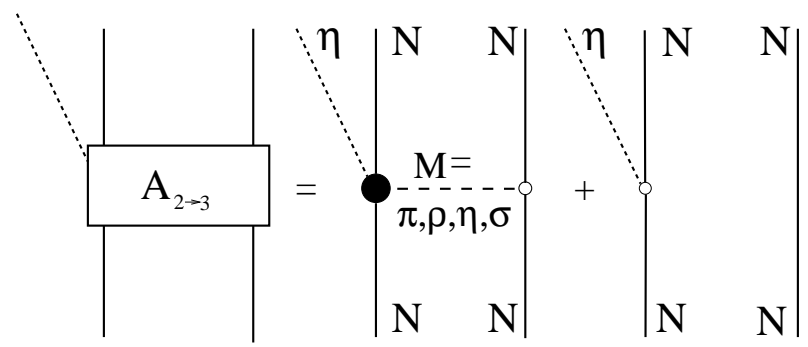

a)

b)

FIG. 1. Production mechanisms for the reaction $N N \rightarrow N N \eta$ taken into account in our model: (a) $\eta$ production via $M N \rightarrow \eta N$ rescattering; (b) direct $\eta$ production.

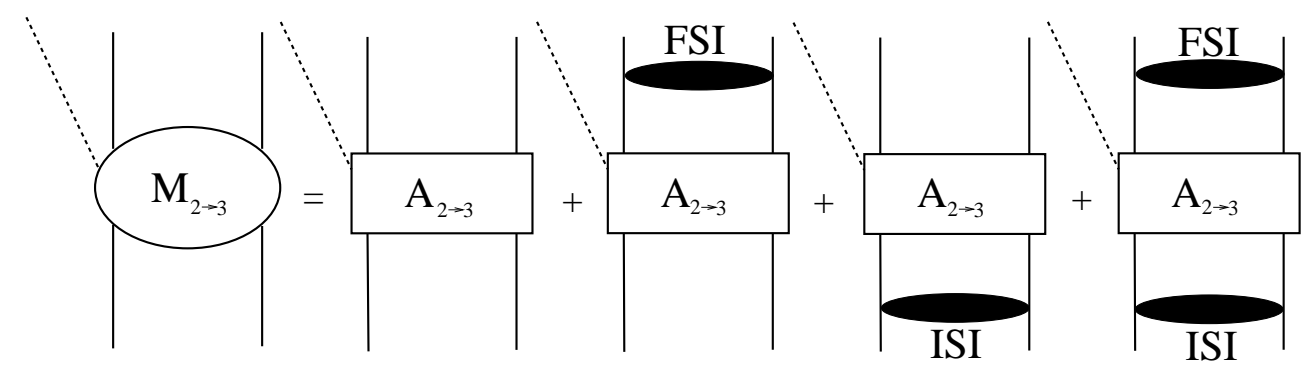

FIG. 2. Diagramatic representation of our DWBA calculation. $A_{2 \rightarrow 3}$ is the elementary $\eta$-production amplitude. The filled ellipses stand for the $N N$ interaction in the final and initial states.

Phase shift (degree)

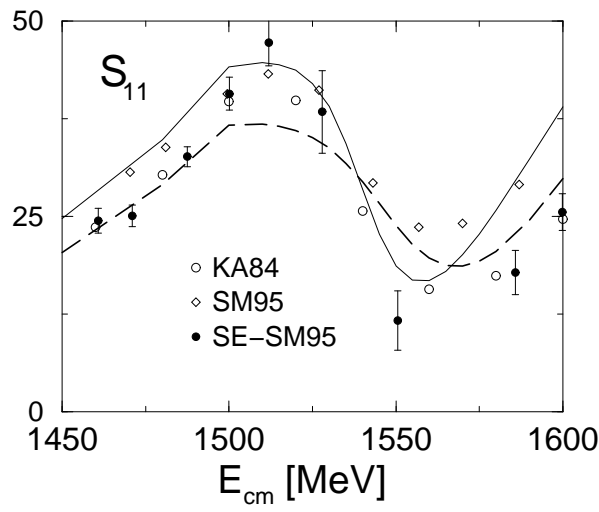

inelasticity $\left(1-\eta^{2}\right)$

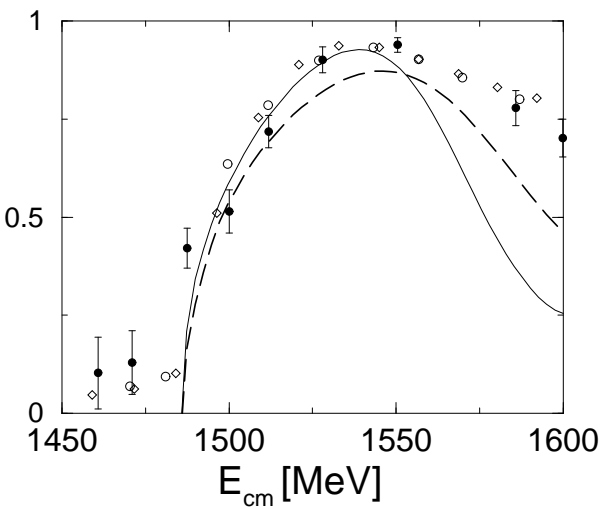

FIG. 3. The $\pi N$ phase shift and inelasticity $1-\eta^{2}$ for the $S_{11}$ partial wave. The dashed curves represent the results of the original $\pi N$ model of Ref. [18,39] whereas the solid lines are the results of the extended model that includes the additional diagrams of Fig. 4. 


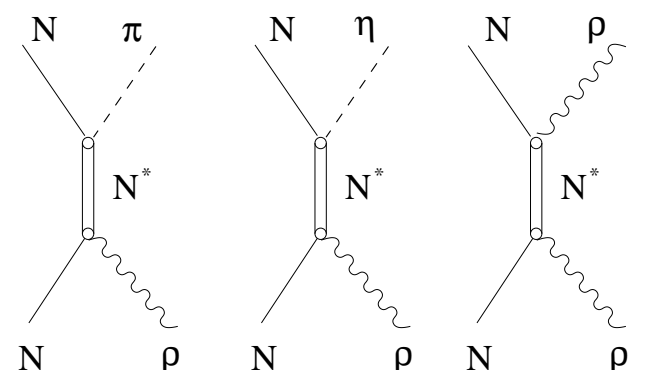

FIG. 4. Additional diagrams containing the $\rho N N^{*}$ coupling which are included in the extended model.

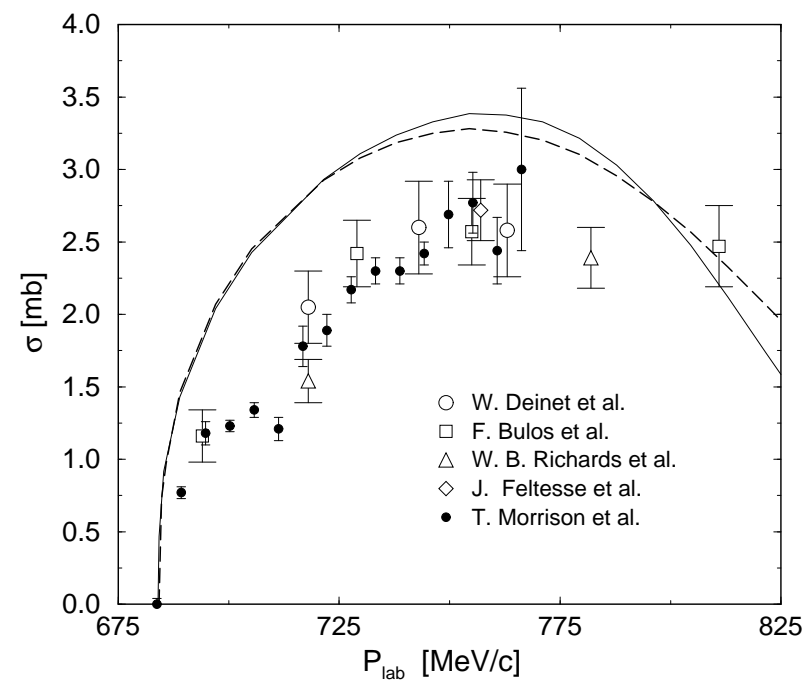

FIG. 5. S-wave cross section for the reaction $\pi^{-} p \rightarrow \eta n$. The dashed curve represents the results of the original $\pi N$ model of Refs. $[18,39]$ whereas the solid line is the result of the extended model that includes the additional diagrams of Fig. 4. Data are taken from Ref. [41]. 

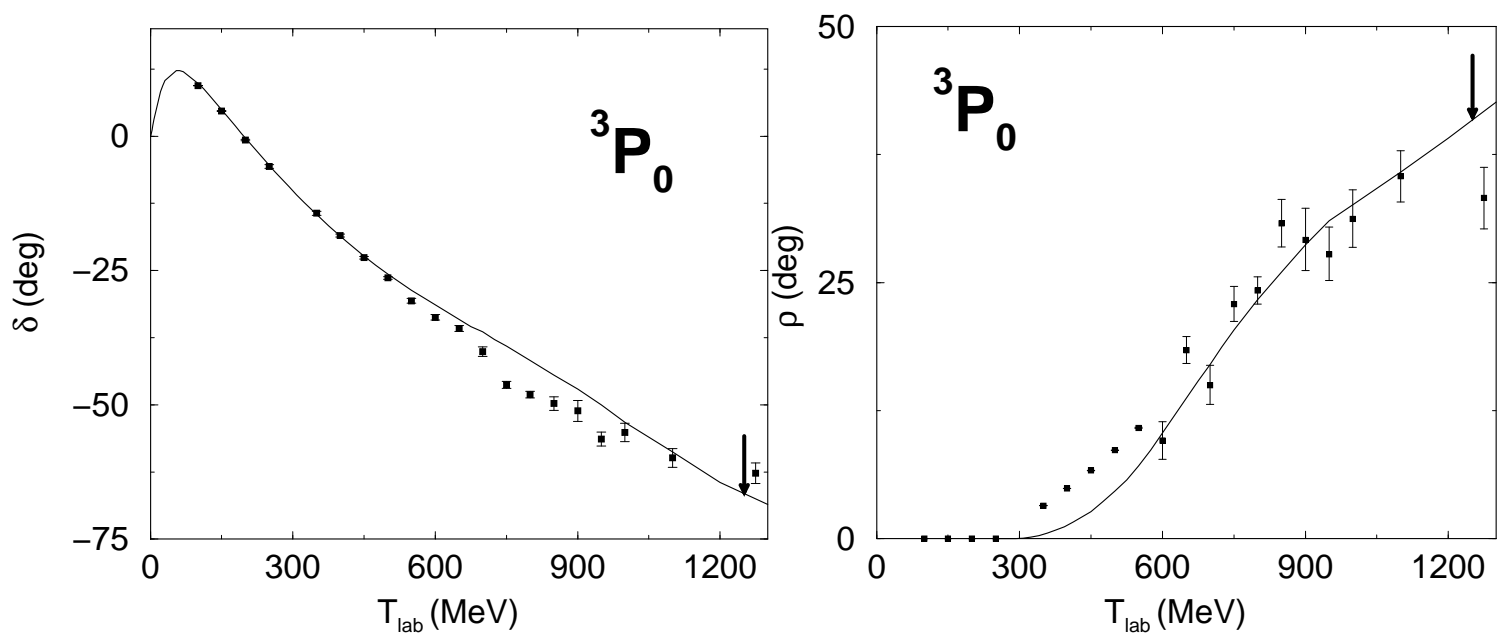

FIG. 6. Phase shift $\delta$ and inelasticity parameter $\rho\left(\eta=\cos ^{2} \rho\right)$ calculated for the ${ }^{3} P_{0}$ partial wave using the CCF model [47]. The squares represent experimental phase shifts, extracted from the SAID library [44] and recalculated from the Arndt-Roper convention [48] to satisfy the condition for the S-matrix: $S=\eta e^{2 i \delta}$. The arrows indicate the $\eta$ production threshold.
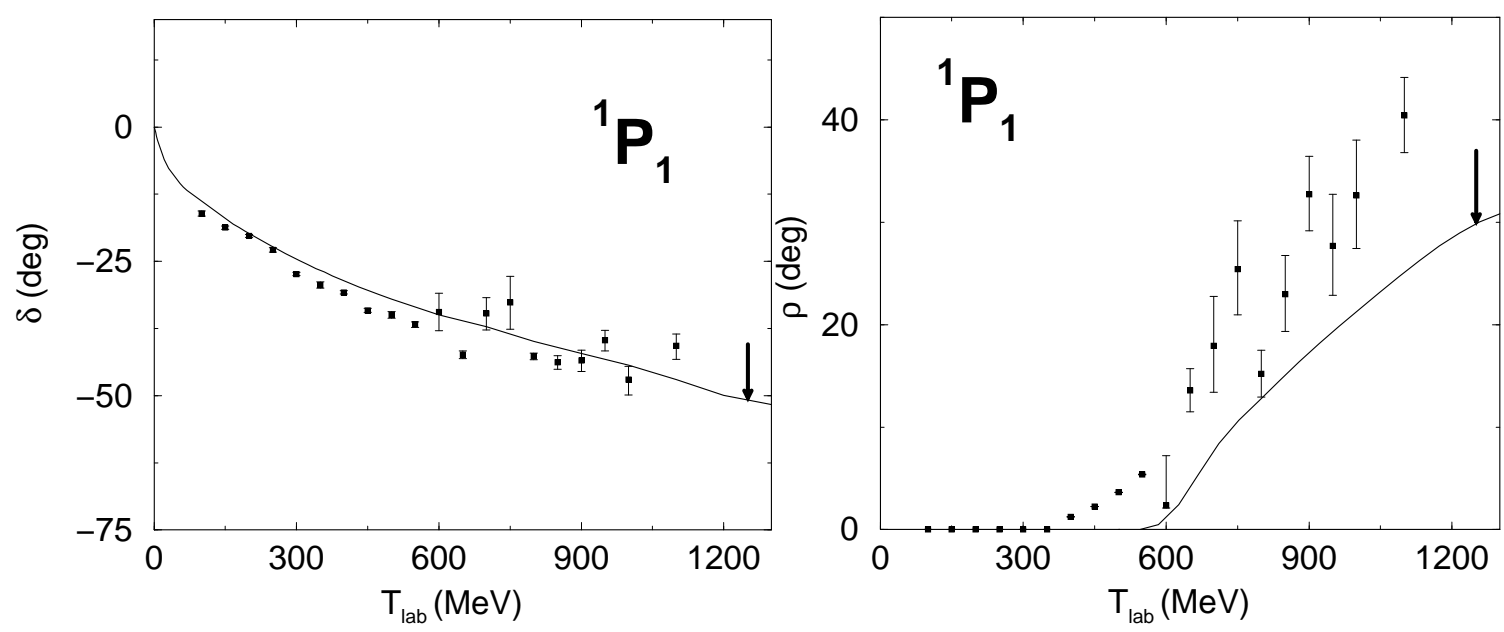

FIG. 7. Phase shift $\delta$ and inelasticity parameter $\rho\left(\eta=\cos ^{2} \rho\right)$ calculated for the ${ }^{1} P_{1}$ partial wave using the CCF model [47]. Same description of squares as in Fig. 6. 

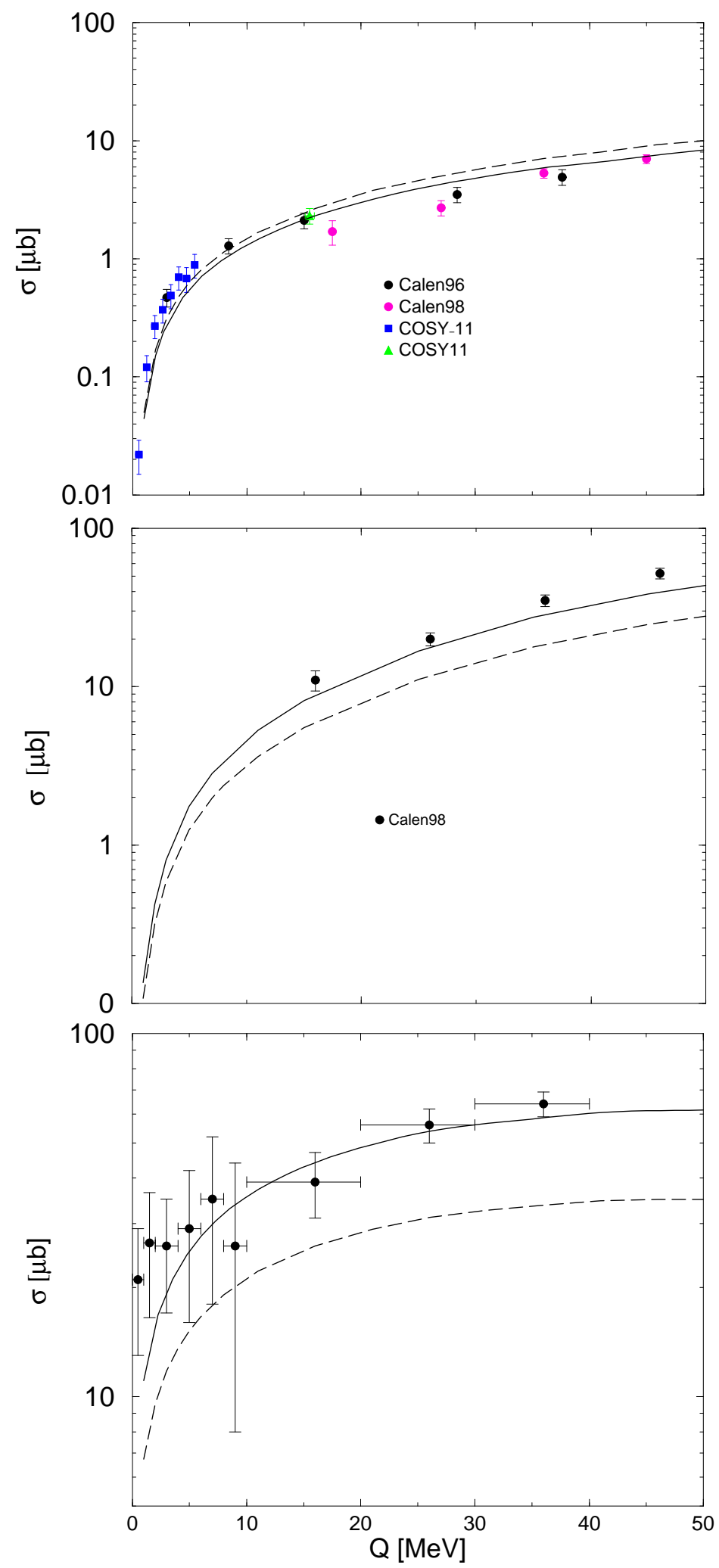

FIG. 8. Total cross sections of the reactions $p p \rightarrow p p \eta$ (top), $p n \rightarrow p n \eta$ (middle) and $p n \rightarrow d \eta$ (bottom). The dashed lines represent the results of our calculation with the original $M N$ model of Ref. [18], whereas the solid lines are based on the extended $M N$ model described and discussed in Sect. II A. Data are taken from Refs. [6,8-12]. 


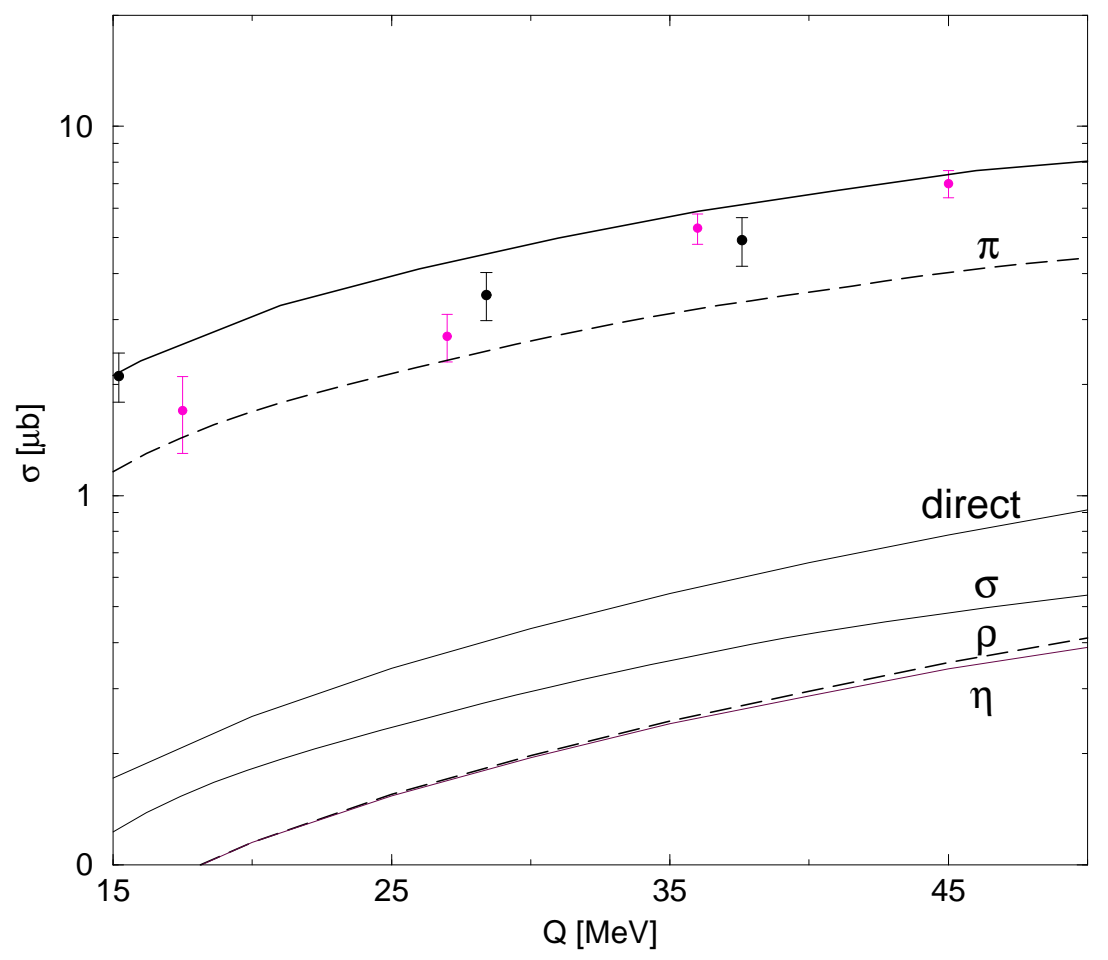

FIG. 9. Contributions of the individual meson exchanges to the reaction $p p \rightarrow p p \eta$. The presented results are based on the extended $M N$ model. The solid line is our full result, i.e. when all contributions are summed up coherently.

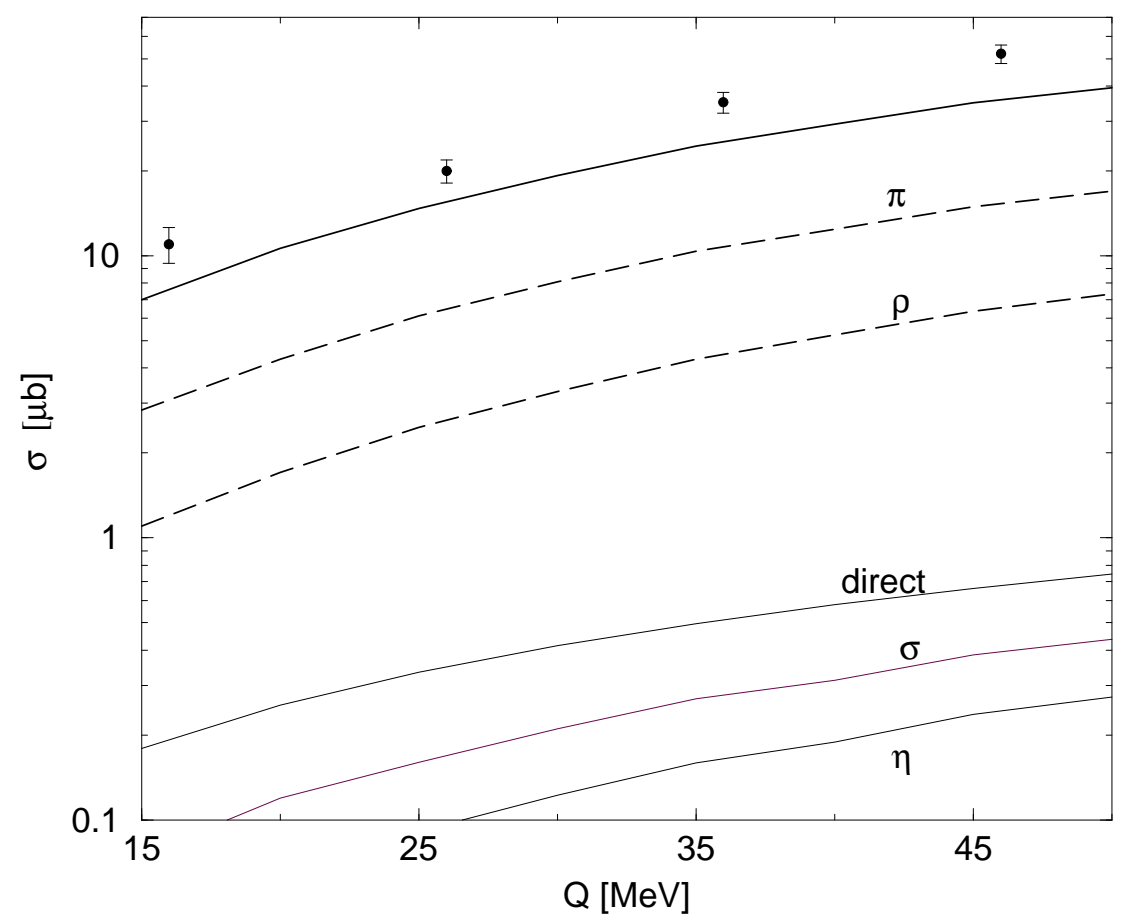

FIG. 10. Contributions of the individual meson exchanges to the reaction $p n \rightarrow p n \eta(I=0)$. The presented results are based on the extended $M N$ model. The solid line is our full result, i.e. when all contributions are summed up coherently. 


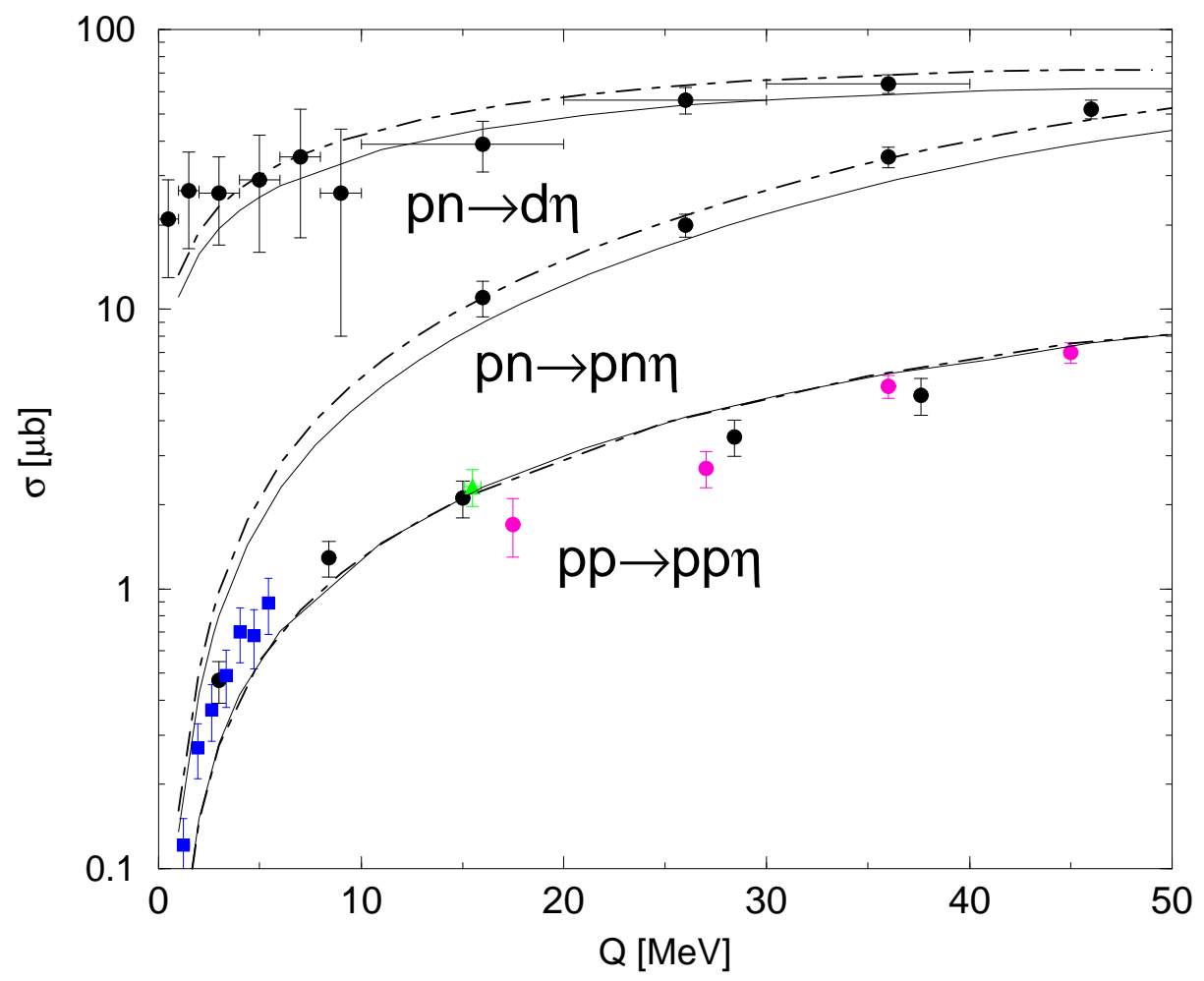

FIG. 11. Total cross sections of the reactions $p p \rightarrow p p \eta, p n \rightarrow p n \eta$, and $p n \rightarrow d \eta$ employing different $N N$ models for the final state interaction. The solid lines represent the results with the CCF $N N$ model [47] whereas the dashed-dotted lines were obtained for the Bonn B model [54]. The calculations are based on the extended $M N$ model. 